\title{
Pulsus Alternans Involving the Right Heart
}

\author{
Kunitake Hashiba, M.D., * Tomoyuki Katayama, M.D., Akira \\ Takahashi, M.D., Akio Ono, M.D., Junshi Fujita, M.D., \\ Shuzo Matsuo, M.D., Nobuo Tajima, M.D., Yoshimi \\ Yamaguchi, M.D., Yoshito Yoshioka, M.D., \\ and Kayuko Mrura, M.D.
}

\begin{abstract}
Alternation of the pulmonary arterial, right ventricular and right atrial pressures was studied in 4 patients with mitral stenosis and severe pulmonary hypertension and in one with postpartum myocardial disease. It was concluded that pulsus alternans in the pulmonary circulation was elicited in close connection with severe right ventricular strain or failure. Premature beat of ventricular origin was one of the most important trigger mechanism initiating pulsus alternans. Contrary to the previous opinion in the literature pulsus alternans following premature beats persists for a rather long time, at least in the presence of pulmonary hypertension. Pulsus alternans was induced in a patient by exercise.
\end{abstract}

DULSUS alternans has been considered a sign of severe ventricular failure and is usually found only in the presence of organic heart diseases, especially in patients with hypertensive and/or ischemic heart disease ${ }^{1 /-3)}$ and aortic stenosis.") Although the fundamental mechanism of pulsus alternans is still to be investigated, several factors ${ }^{4-11)}$ have been known to influence its appearance and severity.

Pulsus alternans is usually recognized in the systemic circulation by palpation and/or auscultation of the heart and peripheral pulse. Only cardiac catheterization can demonstrate the presence of pulsus alternans in the pulmonary circulation and few reports ${ }^{19,12), 13)}$ have been found in the literature concerning this entity. It is the purpose of this paper to report 5 patients in whom pulsus alternans was found in the pulmonary circulation. Four of them had mitral stenosis and severe pulmonary hypertension and the other had postpartum myocardial disease.

\section{Gase Reports}

Case 1. M.S., a 20-year-old man, was admitted to the hospital because of exertional dyspnea on March 3, 1964. Six years before admission, he felt dyspneic

From the First Department of Internal Medicine (Director: Prof. Y. Takaoka), Nagasaki University School of Medicine, Nagasaki.

* Associate Professor of Internal Medicine. 
a.

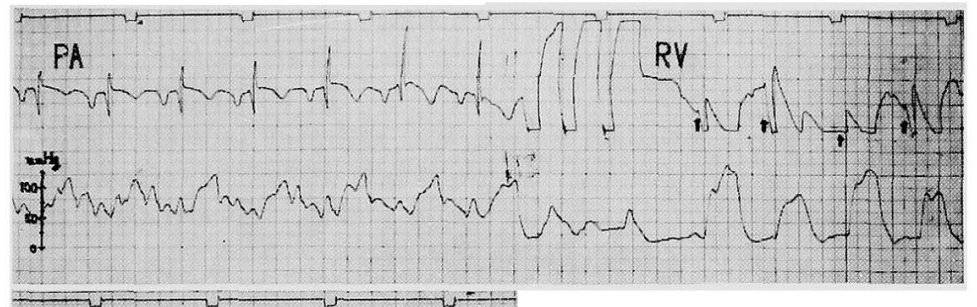

b.

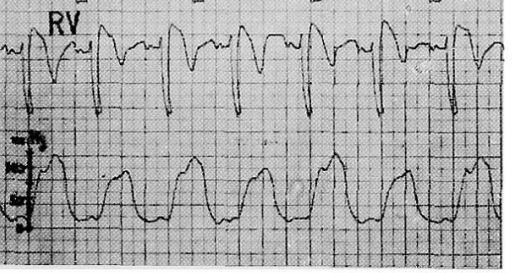

Fig. 1. Pulsus alternans in the right ventricular pressure induced by ventricular premature beats in case 1 .

a) Recorded while withdrawing catheter from the pulmonary artery into the right ventricle. Just when the tip of the catheter fell into the right ventricle, 3 successive premature beats of ventricular origin appeared and were immediately followed by marked pulsus alternans. Four arrows for the ECG tracing indicate the beginning of QRS complex of restored sinus rhythm. EGG taken by catheter electrode. Time interval $1 \mathrm{sec}$.

b) Recorded $1 \mathrm{~min}$. later, showing persistence of the marked alternation. The alternation persisted for about $3 \mathrm{~min}$.

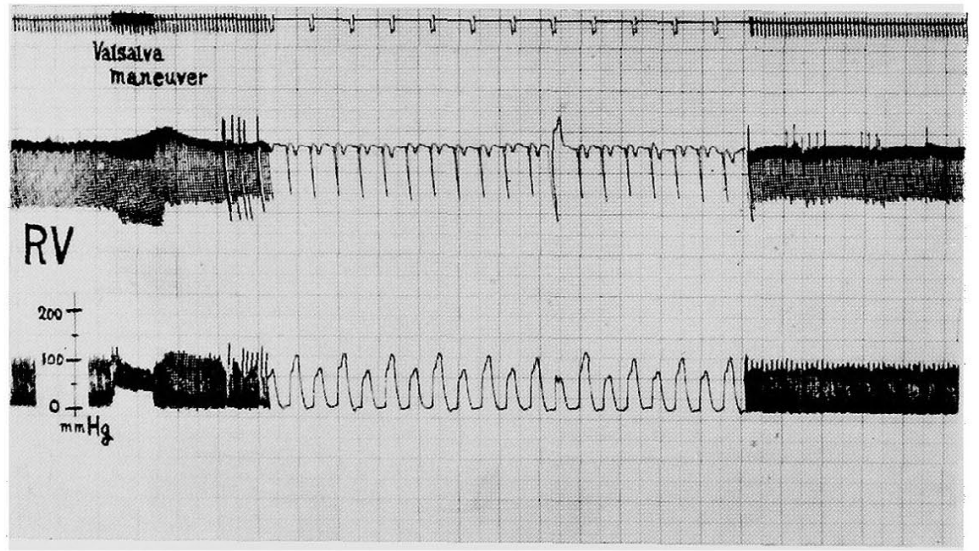

Fig. 2. Another occurrence of pulsus alternans in the right ventricular pressure following ventricular premature beats in case 1. After the Valsalva maneuver several ventricular premature beats appeared and again were followed by marked pulsus alternans which persisted for over $50 \mathrm{sec}$. Slight alternation of $\mathrm{S}$ waves was also found in the middle of the tracing (see Discussion). EGG taken by catheter electrode. Time interval 1 sec. Paper speed was changed twice in this tracing. Black bar on the timer indicates the Valsalva maneuver of $10 \mathrm{sec}$.

while playing baseball, and was told by a doctor that he had heart disease. Since 1960 he worked in tailor shop and sometimes developed exertional dyspnea. 
In September 1963 he had an episode of hemoptysis. In January 1964 he developed edema, dyspnea and orthopnea. On admission the patient was not dyspneic at rest. Respiratory rate was 20, heart rate was 88, and blood pressure was 122 systolic and 84 diastolic. Cardiac sounds and murmur indicated mitral stenosis. Chest X-ray revealed an enlarged heart, a prominent pulmonary artery, and markedly increased vascular markings and fibrosis in the lung fields. Electrocardiogram showed a mitral $\mathrm{P}$ wave and right ventricular hypertrophy.

Cardiac catheterization was performed on March 19, 1964. Pulmonary arterial pressure was 110 systolic and 55 diastolic. Upon withdrawing the catheter from the pulmonary artery into the right ventricle, a short run of 3 successive ventricular premature beats appeared, and immediately after the cessation of the premature beats a marked alternation of the right ventricular pressure started and persisted for about $3 \mathrm{~min}$. (Fig. 1). Although simultaneous recording of the systemic blood pressure was not obtained, pulsus alternans was also found in the radial pulse by palpation. A few minutes later, the Valsalva maneuver was carried out, placing the tip of the catheter in the right ventricle. Seventeen sec. after the maneuver several ventricular premature beats appeared and again were followed by a marked alternation of the right ventricular pressure which persisted for about 1 min. (Fig. 2).

Case 2. K.Y., a 37-year-old housewife, was admitted to the hospital on January

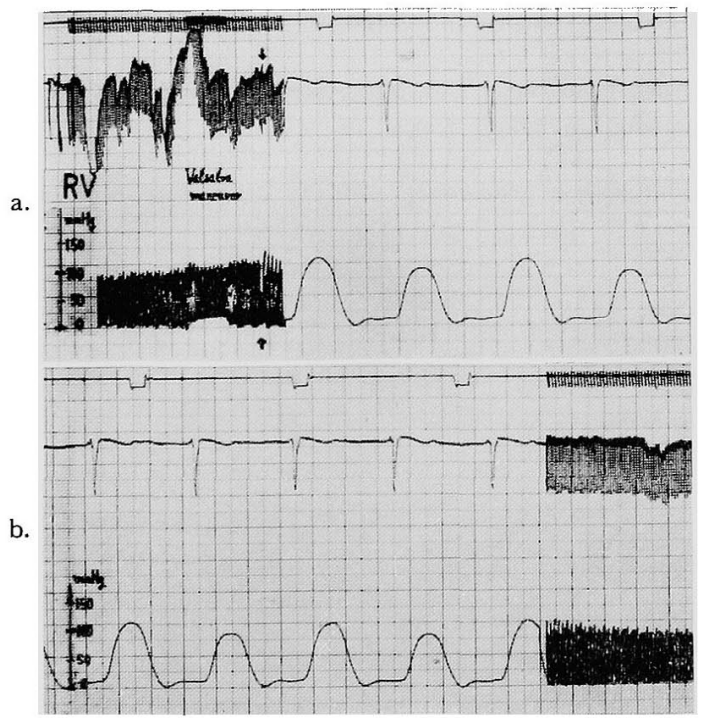

Fig. 3. Exaggeration of pulsus alternans in the right ventricular pressure induced by premature beat in case 2 .

a) Pulsus alternans was found before and throughout the Valsalva mancuver. About $10 \mathrm{sec}$. after the maneuver a premature beat of probably ventricular origin appeared and was followed by a marked exaggeration of the alternation. Pulmonary arterial pressure was increased by the Valsalva maneuver. ECG taken by catheter electrode. Time interval $1 \mathrm{sec}$. Black bar indicates the Valsalva maneuver of $10 \mathrm{sec}$.

b) Recorded $30 \mathrm{sec}$. later, showing gradual disappearance of pulsus alternans as the pulmonaryarterial pressure returned to the previous level. 
14, 1964 because of edema and dyspnea. Two years before admission she noticed exertional dyspnea and sometimes became orthopneic in the night. Thereafter the symptoms were intermittent. On admission the patient was not dyspneic at rest. Respiratory rate was 21, heart rate was 100 , and blood pressure was 166 systolic and 80 diastolic. Cardiac sounds and murmur indicated mitral stenosis. Chest $\mathrm{X}$-ray revealed an enlarged heart and increased pulmonary vascular markings. Electrocardiogram showed a $\mathrm{P}$-sinistrocardiale in $\mathrm{V}_{1}$ and right ventricular hypertrophy.

Cardiac catheterization was performed on February 5, 1964. The pulmonary arterial pressure was 82 systolic and 50 diastolic. Transiently appearing pulsus alternans of slight degree was found in several occasions throughout the catheterization, and also was found just before and during the Valsalva maneuver (Fig.3). About 10 sec. after the Valsalva maneuver a premature beat of probably ventricular origin appeared (indicated by arrows in Fig. 3), immediately after which a marked exaggeration of the already existing pulsus alternans of the right ventricular pressure was found in the tracing. The marked alternation persisted for about $1 \mathrm{~min}$. and gradually diminished as the right ventricular pressure returned to the control level.

The patient died from congestive heart failure in March, 1965.

Case 3. Y. M., a 17-year-old girl, was admitted to the hospital because of exertional dyspnea on November 25, 1963. Seven years before admission the

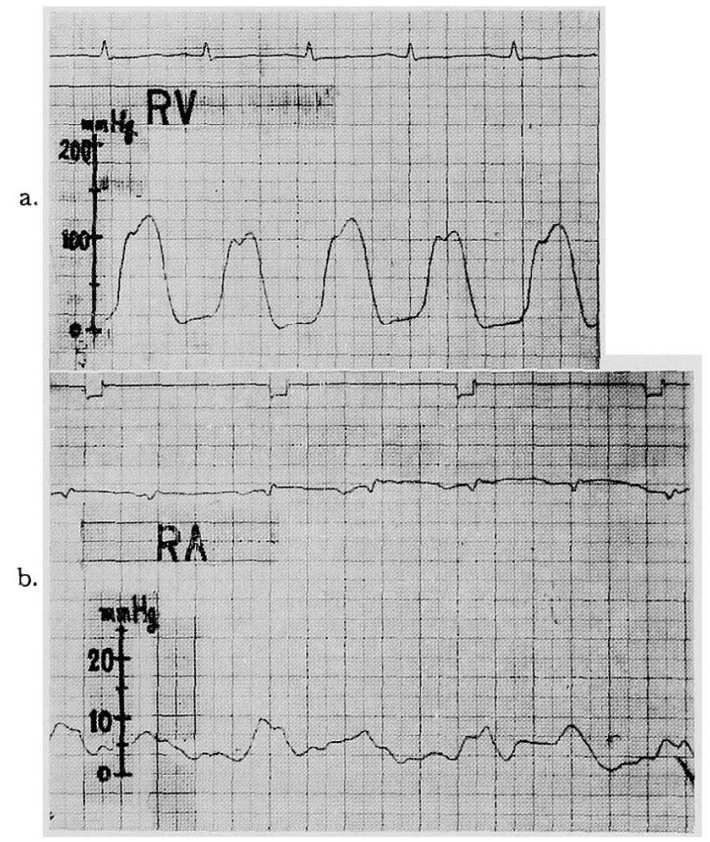

Fig. 4. Pulsus alternans in case 3 ; a) in the right ventricular pressure. The larger beats started at lower end-diastolic pressure than the smaller beats. ECG lead II. Paper speed $50 \mathrm{~mm}$. $/ \mathrm{sec}$. b) in the right atrial pressure. Alternation was found in both ' $a$ ' and ' $v$ ' waves. EGG taken by catheter electrode. Time interval $1 \mathrm{sec}$. 
patient was first told that she had valvular disease. For 2 years she had been treated intermittently with digitalis and with antibiotics because of exertional dyspnea and suspected endocarditis. On admission respiratory rate was 26 , heart rate was 90, and blood pressure was 102 systolic and 64 diastolic. A grade III/VI diastolic murmur was audible at the apex and there was a grade II/VI GrahamSteell murmur at the pulmonic area. The second pulmonic sound was markedly accentuated. Chest X-ray revealed an enlarged heart and increased vascular markings in the lung fields. Electrocardiogram revealed a mitral $\mathrm{P}$ wave and marked right ventricular hypertrophy.

Cardiac catheterization was performed on January 29, 1964. The pulmonary arterial pressure was 130 systolic and 40 diastolic. Immediately after the tip of catheter was drawn back into the right ventricle, multi-focal ventricular premature beats appeared successively for several seconds. A little later, when the arrhythmia subsided, an alternation was found in the right ventricular pressure (Fig. 4 a). In this tracing the diastolic pressure was also alternating, demonstrating the pressure difference of 4 to $6 \mathrm{~mm} . \mathrm{Hg}$ at end-diastole. An alternation of the pressure was also recorded from the right atrium shortly after that of the right ventricle (Fig. 4 b). The alternation of the ' $v$ ' wave was more significant than that of the ' $a$ ' wave.

Case 4. S. T., a 31-year-old man, was admitted to the hospital because of ex-

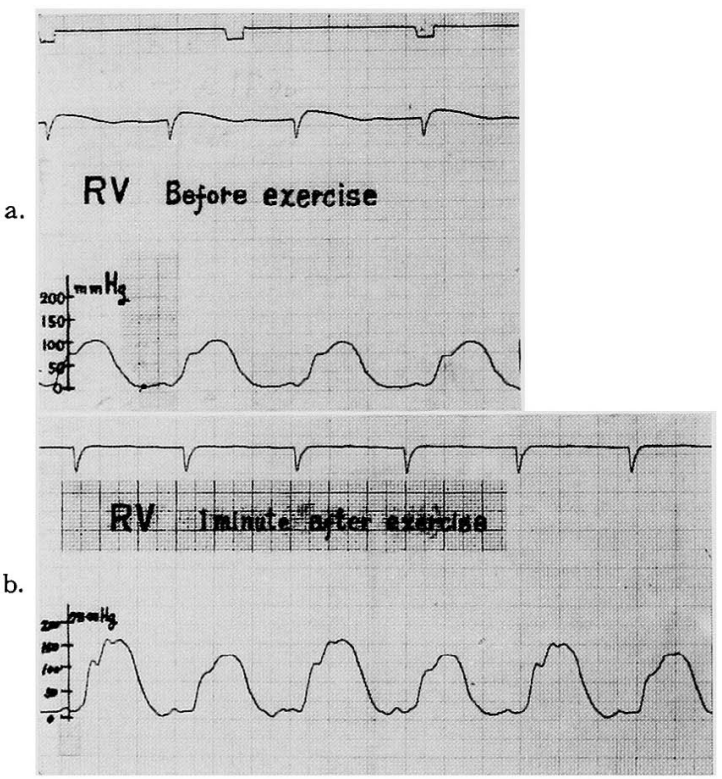

Fig. 5. Pulsus alternans in the ventricular pressure induced by exercise in case 4 .

a) Before exercise. No alternation was found. Duration of the exercise was $3 \mathrm{~min}$. ECG taken by catheter electrode. Time interval $1 \mathrm{sec}$.

b) Recorded $1 \mathrm{~min}$. after the exercise. Alternation of the right ventricular pressure appeared during the exercise as the pressure was increased, and it became more marked after the exercise. The alternation was abolished in about 7 min. after the exercise as the right ventricular pressure returned to the previous level. 
ertional dyspnea on May 11, 1963. Three years before admission he first noticed dyspnea when walking with his children, and therafter he frequently suffered from exertional dyspnea, especially on walking up hill or walking in a hurry. On admission the patient was not dyspneic but slightly cyanotic about his cheeks and lips. Respiration was normal, heart rate was 80 , and blood pressure was 134 systolic and 92 diastolic. The liver was palpable 2 fingerbreadths below the right costal margin with slight tenderness. Cardiac sounds and murmur indicated mitral stenosis. Electrocardiogram revealed right ventricular hypertrophy.

Cardiac catheterization was performed on May 29, 1963, and revealed severe pulmonary hypertension. An exercise test was carried out during the catheterization. Right ventricular systolic pressure was $105 \mathrm{~mm}$. $\mathrm{Hg}$ before the exercise and was elevated up to $165 \mathrm{~mm} . \mathrm{Hg}$ during the exercise, and gradually returned to the previous level in about $7 \mathrm{~min}$. An alternation of the right ventricular pressure appeared during the exercise and became more marked after the exercise was stopped (Fig. 5). It disappeared gradually as the increased right ventricular pressure returned to the pre-exercise level. Simultaneous recording of the systemic blood pressure was not obtained. At the end of the catheterization a femoral puncture was performed and revealed no alternation of the pressure at rest. Pulsus alternans of the systemic pressure was found immediately following the Valsalva maneuver and disappeared in about $10 \mathrm{sec}$.

The patient died from congestive heart failure in November, 1964.

Case 5. K. H., a 36-year-old farmer's wife, was admitted to the hospital because of dyspnea, for the first time on September 12, 1962. The patient had experienced 5 deliveries and had no history of heart disease previously. Slight edema was noticed in the lower extremities during her last pregnancy and also after her last delivery on May 17, 1962. She returned to her farm work in August of the same year, and then exertional dyspnea appeared. Since the beginning of September the patient was orthopneic continuously. On admission the patient was orthopneic and respiration was 30 per min. Heart rate was 126 and blood pressure was 140 systolic, 84 diastolic. A grade II/VI systolic murmur and a diastolic gallop rhythm were audible at the apex. The liver was palpable 3 fingerbreadths below the right costal margin. Chest X-ray showed globular cardiomegaly and marked pulmonary congestion. Electrocardiogram revealed sinus tachycardia, normal mean electrical axis, inverted $\mathrm{T}$ waves and ST depression in I, II, III, a $V_{F}$ and $V_{4}$ through $V_{6}$. Postpartum myocardial disease was suspected and rapid digitalization was started which improved the symptoms in a week. On the third hospital day pulsus alternans was noticed in the radial pulse and was confirmed by femoral puncture. The patient was discharged on October 15,1962 . The patient did not regularly continue digitalis and was readmitted to the hospital because of increasing cardiac decompensation on June 4, 1963. On the second admission respiratory rate was 34 and heart rate was 110 . The liver was 3 fingerbreadths palpable with tenderness, and there was moderate pedal edema. Electrocardiogram revealed increased ST depression as compared with that of the first admission. Cedilanid was given intravenously and the symptoms were much improved in a month.

Cardiac catheterization was performed on September 4, 1963. Pulsus alternans was recorded from the pulmonary artery (Fig. $6 \mathrm{a}$ ), right ventricle (Fig. $6 \mathrm{~b}$ ), and right atrium (Fig. $6 \mathrm{c}$ ). There was no pulmonary hypertension and the range 


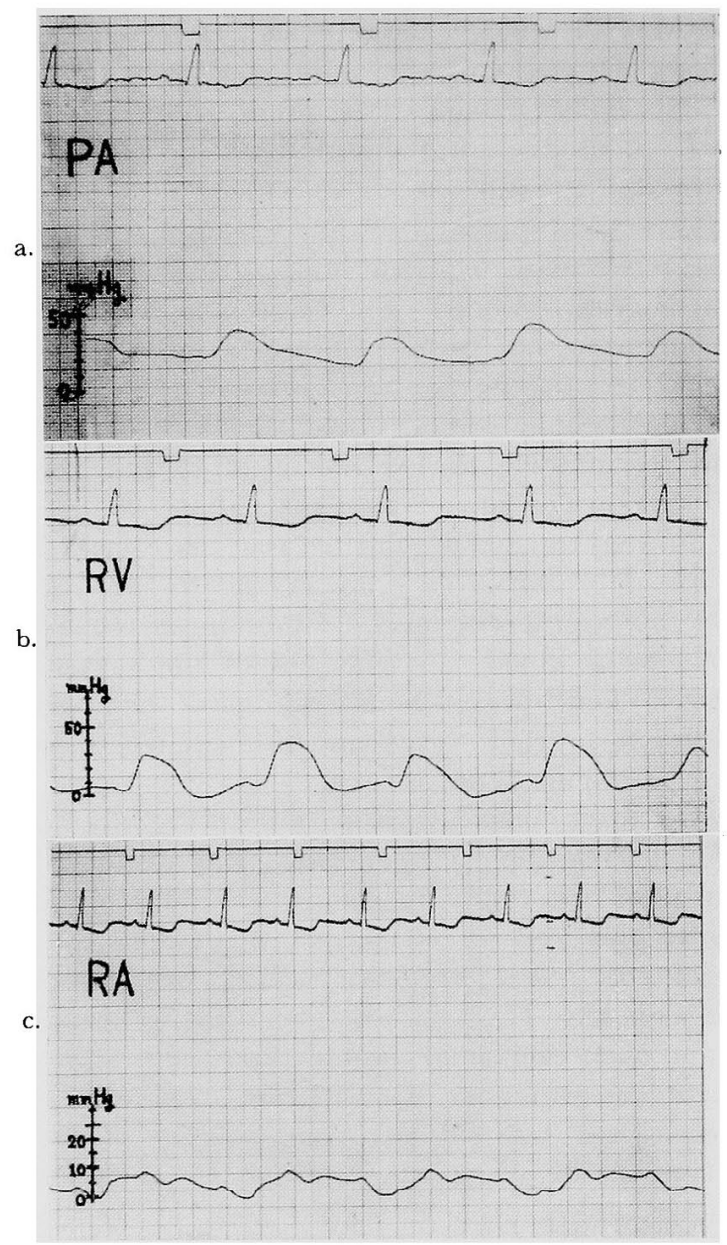

Fig. 6. Pulsus alternans in case 5 ; a) in the pulmonary arterial pressure. ECG lead II. Time interval 1 sec. b) in the right ventricular pressure. Alternation was also found in early diastole but not at end-diastole. c) in the right atrial pressure. Alternation was found in both ' $a$ ' and ' $v$ ' waves. Paper speed $25 \mathrm{~mm}$. sec.

of the alternation of the pulmonary arterial pressure was $6 \mathrm{~mm} . \mathrm{Hg}$. In the tracing of the right ventricular pressure an alternation was also found in early diastole but not at end-diastole. In the atrial pressure the alternation was more marked in the ' $v$ ' waves than in the ' $a$ ' waves. Although simultaneous recording of the pressure was not obtained, pulsus alternans was felt by palpation of the radial pulse.

\section{Discussion}

Although pulsus alternans in the lesser circulation is not considered an extreme rarity, only a few reports ${ }^{10), 21), 13)}$ can be found on this subject. In 
other reports ${ }^{3 i, 14), 15)}$ pulsus alternans in the pulmonary circulation was only incidentally described. The first extensive study of pulsus alternans in the lesser circulation in man was that of De Rabago, Kohout and Katz in a patient with atrial septal defect. ${ }^{12}$ ) A series of patients in whom pulsus alternans was observed in the pulmonary circulation was reported by Ferrer, Harvey, Cournand and Richards. ${ }^{10)}$ Among 21 patients included in their series 6 showed pulsus alternans in the pulmonary circulation alone, another 6 in the systemic circulation alone, and the remaining 9 in both circulations. By simultaneous recordings of the pressure in both circulations they showed that alternation of the pressure could occur independently in either the greater or the lesser circulation without appearing in the other. $\mathrm{McIntosh}^{13)}$ reported a patient in whom discordant pulsus alternans was observed in both circulations. In the series reported here alternation of the pressure was found in the pulmonary circulation in 5 patients, including 4 with mitral valvular disease and 1 with postpartum myocardial disease. Although in some patients of our series alternation was not found in the systemic circulation, no evidence could be presented concerning the dependency and synchronicity of pulsus alternans in 2 circulations because of the lack of simultaneous pressure rccordings.

It would be noteworthy that almost all the patients reported in the literatures in whom pulsus alternans was found in the pulmonary circulation had right-sided heart disease or pulmonary hypertension. ${ }^{101,12), 13)}$ In the series of Ferrer and associates ${ }^{10)} 6$ patients in whom pulsus alternans was found in the pulmonary circulation alone had rheumatic or hypertensive heart disease, and in 5 of them marked pulmonary hypertension was demonstrated. Moderate pulmonary hypertension was also demonstrated in 7 out of 9 patients of their series in whom alternation of the pressure was observed in both circulations. In the series reported here 4 patients had mitral valvular disease and severe pulmonary hypertension and the other patient with postpartum myocardial disease had clinical signs of right-sided failure. In all our patients physical activity was more or less restricted because of exertional dyspnea, and 2 patients died from congestive heart failure in 2 years after the catheterization. It could be deduced, therefore, that pulsus alternans in the pulmonary circulation implied right ventricular myocardial insufficiency resulting either from direct involvement of the right ventricular myocardium or from right ventricular strain due to pulmonary hypertension, and that it probably indicated a poor prognosis.

Pulsus alternans is usually found in the systolic period of arterial or ventricular pressure tracings. However, minor alternations in the diastolic pressure might not be detected by routine pressure monitoring techniqucs. The 
effect of changing end-diastolic pressure upon ventricular systolic pressure presents an interesting problem in the mechanism of pulsus alternans. De Rabago and associates ${ }^{8}$ observed in their patient that the stronger beats of pulsus alternans started at higher end-diastolic pressure of the right ventricle than the weaker beats. Ferrer and associates ${ }^{10}$ noted that in none of the patients of their series was alternation found either in the diastolic phase of the right ventricular pressure or in the right atrial pressure. Mitchell and associates $^{16)}$ concluded from the experimental evidence that the weak beat was initiated from a shorter end-diastolic fiber length than is the strong beat, whether the end-diastolic pressure is lower, the same, or higher. By means of serial biplane angiography utilizing injections into the left ventricle Gleason and Braunwald ${ }^{17}$ observed in a patient of rheumatic mitral disease that pulsus alternans was accompanied by concordant alternation of the left ventricular end-diastolic volume; i.e., the larger end-diastolic volumes resulted in larger stroke volumes and higher systolic pressures than did the smaller end-diastolic volumes. In 2 patients of our series, alternation was also found in the pressure curves of the right atrium and in the diastolic period of the right ventricle. In the case 3 , the right ventricular diastolic pressure was alternating and the larger beats started at lower end-diastolic pressures than the smaller beats. In the case 5, the early diastolic pressure was alternating but the end-diastolic pressure showed no alternation. In both cases the right atrial pressure was also alternating, showing alternation in both ' $a$ ' and ' $v$ ' waves. Alternation in ' $v$ ' waves was more marked than in ' $a$ ' waves in both cases. From the results observed in our cases it is difficult to believe that alternation of the ventricular diastolic pressure plays a primary role in the mechanism of pulsus alternans.

Pulsus alternans is generally more or less a labile condition of the circulation. Exercise is one of the factors which influence the appearance, disappearance and severity of pulsus alternans. It sometimes induces or intensifies $^{10)}$ and sometimes abolishes or diminishes ${ }^{9)}$ pulsus alternans. In the case 4 of our series an exercise induced pulsus alternans in the right ventricular pressure, as it was markedly elevated during and after the exercise. Ferrer and associates ${ }^{10)}$ noted 2 rheumatic patients in whom pulsus alternans appeared in association with a rise in the pulmonary arterial pressure during exercise.

The other important factor in our series that induced or intensified pulsus alternans was the premature beat. Pulsus alternans is sometimes induced or exaggerated by premature beat of ventricular origin, but in that case it usually disappears or diminishes within 10 beats after the premature beats. $\left.\left.{ }^{2,}, 6\right), 7\right), 15$ ) In the opinion of Ferrer and associates ${ }^{10}$ it is difficult to believe that post- 
extrasystolic pulsus alternans goes on for as long as 10 beats. In the case 2 of our series an alternation of the right ventricular pressure was found just before and during the Valsalva maneuver. About $10 \mathrm{sec}$. after the strain of the maneuver a premature beat of probably ventricular origin (indicated by arrows in Fig. 3) appeared and was followed by a marked exaggeration of the already existing pulsus alternans. The exaggeration of pulsus alternans persisted for about 30 sec., and pulsus alternans disappeared as the right ventricular pressure returned to the control level. In this case premature beat was the trigger mechanism for the exaggeration, and an increase in the pulmonary arterial pressure following the maneuver might be a factor in maintaining the exaggeration. In the case 1, 3 successive ventricular premature beats appeared just when catheter tip was drawn back from the pulmonary artery into the right ventricle, and were followed by a marked alternation of the right ventricular pressure (Fig. 1). Pulsus alternans persisted for about $3 \mathrm{~min}$. In the same patient several ventricular premature beats appeared about $15 \mathrm{sec}$. after the Valsalva maneuver, and again were followed by a marked alternation of the right ventricular pressure which persisted for over $1 \mathrm{~min}$. (Fig. 2). In our observation of the cases, premature beats could induce pulsus alternans which persisted for a rather long time, at least in the presence of pulmonary hypertension.

It should be noted that in the latter tracing of the case 1 (Fig. 2) a slight alternation of $\mathrm{S}$ waves of intracavitary electrocardiogram was also found coincidentally with the mechanical alternation. Coexistence of electrical and mechanical alternation is a very rare condition although it has been postulated that the underlying mechanism of the two alternations is fundamentally the same and that one cannot be present without the other. ${ }^{15}$ Ellis $^{18)}$ found coexisting electrical alternation in one-fourth of the dogs in which mechanical alternation was induced by methoxamine hydrochloride and suggested that the mechanism of mechanical alternation is probably independent from that of electrical alternation. Unfortunately, simultaneous recording of conventional electrocardiogram was not obtained in our patient. Therefore, the alternation of $\mathrm{S}$ waves shown in Fig. 2 might only imply alternating position of catheter tip within the right ventricle.

\section{SUMMARY}

Pulsus alternans involving the right side of the heart was studied in 5 patients. Four of 5 patients had mitral stenosis with severe pulmonary hypertension and the other had postpartum myocardial disease with clinical signs indicating both left and right heart failure. It was concluded that 
pulsus alternans in the pulmonary circulation was elicited in close connection with severe right ventricular strain or failure.

Premature beat of ventricular origin was one of the most important trigger mechanism initiating pulsus alternans. Contrary to the previous opinion in the literature pulsus alternans following premature beats could persist for a rather long time, at least in the presence of pulmonary hypertension. Pulsus alternans was induced in a patient by exercise as the pulmonary hypertension was exaggerated.

Alternation of the right ventricular diastolic and right atrial pressures was found in 2 patients in addition to that of the right ventricular systolic pressure. However, it could not be considered that the atrial or right ventricular diastolic alternation played an important role in the genesis of pulsus alternans in the pulmonary circulation.

\section{REFERENCES}

1. White, P.D.: Heart Disease, The Macmillian Company, New York, 1951.

2. Friedberg, C. K.: Diseases of the heart, W. B. Saunders Company, Philadelphia, 1956.

3. Littmann, D.: Circulation $27: 280,1963$.

4. Cooper, T., Braunwald, E., and Morrow, A. G.: Circulation 18: 64, 1958.

5. Green, H. D. : Am. J. Physiol. $114: 407,1936$.

6. Wiggers, C.J.: Circulatory dynamics. Modern medical monographs, No. 4, Grune \& Stratton, New York, 1952.

7. Friedman, B., Daily, W. M., and Sheffield, R. S. : Circulation 7: 864, 1953.

8. Ryan, J. M., Schieve, J. F., Hull, H. B., and Oser, B. M. : Girculation 12:60, 1955.

9. Ryan, J. M., Schieve, J. F., Hull, H. B., and Oser, B. M. : Circulation 14: 1099, 1956.

10. Ferrer, M. I., Harvery, R. M., Cournand, A., and Richards, D. W.: Circulation 14: 163, 1956.

11. Ellis, C. H. : Am. J. Physiol. 199: 167, 1960.

12. De Rabago, P., Hohout, F. W., and Katz, L. N.: Am. Heart J. 49: 472, 1955.

13. McIntosh, H. D.: Circulation $21: 214,1960$.

14. Weisse1, W., Salzmann, F., and Vetter, H. : Brit. Heart J. 14: 47, 1952.

15. Littmann, D.: Am. J. Cardiol. 14: 420, 1964.

16. Mitchell, J. H., Sarnoff, S. J., and Sonnenblick, E H. : J. Clin. Invest. 42: 55, 1963.

17. Gleason, W. L. and Braunwald, E.: Circulation 25: 841, 1962.

18. Ellis, C. H. : Am. J. Physiol. 198 : 327, 1960. 